International Mathematical Forum, 2, 2007, no. 29, 1415 - 1420

\title{
On Multiplication Modules
}

\author{
Ünsal Tekir \\ Marmara University, Department of Mathematics \\ Ziverbey, Göztepe-İstanbul, Turkey \\ utekir@marmara.edu.tr
}

\begin{abstract}
Let $R$ be a commutative ring with identity and $M$ be a unital $R$-module. Then $M$ is called a multiplication module provided for every submodule $N$ of $M$ there exists an ideal $I$ of $R$ such that $N=I M$. Our objective is to investigate properties of prime and semiprime submodules of multiplication modules.
\end{abstract}

Mathematics Subject Classification: 13C05, 13C13

Keywords: Multiplication modules, Prime submodules, Semiprime submodules

Throughout this paper all rings will be commutative with identity and all modules will be unitary. Let $R$ be a ring and $M$ be a unital $R$-module. For any submodule $N$ of $M$, we define $(N: M)=\{r \in R: r M \subseteq N\}$. A submodule $N$ of $M$ is called prime if $N \neq M$ and whenever $r \in R, m \in M$ and $r m \in N$, then $m \in N$ or $r \in(N: M)$. A submodule $N$ of $M$ is called semiprime if $N \neq M$ and whenever $r \in R, m \in M$, and $r^{n} m \in N$ for some positive integer $n$, then $r m \in N$. In recent years, prime and semiprime submodules have attracted a good deal of attention; see, for example [2-5].

An $R$-module $M$ is called a multiplication module provided for each submodule $N$ of $M$ there exists an ideal $I$ of $R$ such that $N=I M$. We say that $I$ is a presantation ideal of $N$. Clearly, every submodule of $M$ has a presantation ideal if and only if $M$ is a multiplication module. Let $N$ and $K$ be submodules of a multiplication with $N=I_{1} M$ and $K=I_{2} M$ for some ideals $I_{1}$ and $I_{2}$ of $R$. The product $N$ and $K$ denoted by $N K$ is defined by $N K=I_{1} I_{2} M$. Then by [6,theorem 3.4], the product of $N$ and $K$ is independent of presentation of $N$ and $K$. Note that this definition is different from the definition of ordinary ideal multiplication. Indeed, let $R=Z$ be the ring of integers, and let $M=2 Z$ and $N=K=4 Z$. Then $N K$ is $16 Z$ by the usual definition and is $8 Z$ by the our definition. Moreover, for $a, b \in M$, by $a b$ we mean the product 
of $R a$ and $R b$. Clearly, $N K$ is a submodule of $M$ and $N K \subseteq N \cap K$ see,for example, $[[7]-[9]$.

The purpose of this paper is to introduce interesting and useful properties of prime and semiprime submodules of multiplication modules.

Theorem 1. Let $M$ be a non-zero $R$-module. Then, $M$ is a faithful $R$-module ( not necessarily multiplication) such that every proper submodule is prime if and only if $R$ is a field.

Proof. $\Leftarrow:$ Clear.

$\Rightarrow$ : Suppose that $R$ is not field. Note that the zero submodule of $M$ is prime. Then $R$ is a domain and $M$ is a torsion-free $R$-module. Because $R$ is not field, $M$ is not simple. Let $R m$ be a proper non-zero submodule of $M$. Assume that $0 \neq a$ is not invertible element of $R$. Then $R a m$ is prime so that $a M$ is contained in Ram ( which gives contradiction $M=R m$ ) or $m$ belongs to $\operatorname{Ram}$ (which gives $R a=R$, a contradiction)

Corollary 2. Let $M$ be a faithful multiplication $R$-module. Then, $M$ is simple if and only if every proper submodule of $M$ is prime.

Proposition 3. Let $M$ be a multiplication $R$-module and $N_{1}, N_{2}, \ldots, N_{k}$ be submodules of $M$.Let $N$ be prime submodule of $M$. Then the following statements are equivalent.

(i) $\quad N_{j} \subseteq N$ for some $j$ with $1 \leq j \leq k$.

(ii) $\bigcap_{i=1}^{k} N_{i} \subseteq N$

(iii) $\prod_{i=1}^{k} N_{i} \subseteq N$

Proof. $(i) \Rightarrow(i i)$ : Clear.

$(i i) \Rightarrow($ iii $)$ : Since $\prod_{i=1}^{k} N_{i} \subseteq \bigcap_{i=1}^{k} N_{i}, \prod_{i=1}^{k} N_{i} \subseteq N$ by $(i i)$.

$($ iii $) \Rightarrow(i)$ : We have $N_{i}=I_{i} M$ for some ideals $I_{i}(1 \leq i \leq k)$ of $R$. Then $N_{1} N_{2} \ldots N_{k}=I_{1} I_{2} \ldots I_{k} M \subseteq N$ and so $I_{1} I_{2} \ldots I_{k} \subseteq(N: M)$. Since $(N: M)$ is a prime ideal of $R, I_{j} \subseteq(N: M)$ for some $j(1 \leq j \leq k)$. Therefore, $N_{j}=I_{j} M \subseteq$ $N$ for some $j(1 \leq j \leq k)$.

Definition 1. Let $M$ be a multiplication $R$-module. A nonempty subset $S^{*}$ of $M$ is said to be multiplicatively closed if $m n \cap S^{*} \neq \varnothing$ whenever $m, n \in S^{*}$.

Proposition 4. Let $M$ be a multiplication $R$-module. Then, a proper submodule $N$ of $M$ is prime if and only if $M \backslash N$ is a multiplicatively closed. 
Proof. Let $N$ be a prime submodule of $M$ and let $a, b \in M \backslash N$. Since $N$ is prime, $a b \nsubseteq N$. Then $a b \cap(M \backslash N) \neq \varnothing$. Conversely, let $a, b \notin N$. Then $a, b \in$ $M \backslash N$. Since $M \backslash N$ is a multiplicatively set, $a b \cap(M \backslash N) \neq \varnothing$. Therefore, $a b \nsubseteq N[$ see, 6$]$.

Theorem 5. Let $M$ be a multiplication $R$-module. Let $A$ be a submodule of $M$ and let $S^{*}$ be a multiplicatively closed set in $M$ such that $A \cap S^{*}$ is empty. Then there is a submodule $N$ of $M$ which is maximal with respect to the properties that $A \subseteq N$ and $N \cap S^{*}$ are empty. Furthermore, $N$ is prime submodule of $M$.

Proof. Let $H$ be the set of all submodules $B$ of $M$ such that $A \subseteq B$ and $B \cap S^{*}$ is empty; $H$ is not empty since $A \in H$. By Zorn's Lemma, $H$ has a maximal element $N$. To show that $N$ is prime, suppose that it is not and let $a b \subseteq N$, $a \notin N, b \notin N$ where $a, b \in M$. Then $N \varsubsetneqq N+R a$ and $N \varsubsetneqq N+R b$ so there are elements $s, t \in S^{*}$ such that $s \in N+R a$ and $t \in N+R b$. Hence $s t \cap S^{*} \neq \varnothing$ and $s t \subseteq(N+R a)(N+R b) \subseteq N$, which contradicts the fact that $N \cap S^{*}$ is empty.

Definition 2. Let $M$ be a multiplication module. $A$ zero divisor in $M$ is an element $0_{M} \neq a \in M$ for which there exists $b \in M$ with $b \neq 0_{M}$ such that $a b=R a R b=0_{M}$.

Theorem 6. Let $M$ be a multiplication $R$-module. Let $N$ be a submodule of $M$ such that $N \neq M$. Then, $N$ is prime if and only if $M / N$ has no zero divisor.

Proof. Suppose that $N$ be a prime submodule of $M$. Since $M$ is a multiplication module, $M / N$ is a multiplication module[see 6 , Theorem 3.21]. Let $a \in M$ be such that the element $0_{M / N} \neq \bar{a}=a+N$ in $M / N$ is zero divisor, so that there exists $b \in M$ such that $0_{M / N} \neq \bar{b}=b+N$ and $\bar{a} \bar{b}=0_{M / N}$. Let $I$ and $J$ be presentation ideals $\bar{a}$ and $\bar{b}$,respectively. Then $\bar{a} \bar{b}=(I J) M / N=N$ and so $a b \subseteq N$. Since $N$ is prime, $a \in N$ or $b \in N$. This is a contradiction. Conversely, let $M / N$ has no zero divisor. Let $a b \subseteq N$ where $a, b \in M$. Then $\bar{a} \bar{b}=0_{M / N}$. Since $M / N$ has no zero divisor, $\bar{a}=0_{M / N}$ or $\bar{b}=0_{M / N}$. Therefore, $a \in N$ or $b \in N$.

Definition 3. Let $M$ be a multiplication $R$-module and let $N$ be a submodule of $M$. Then

(i) $N$ is called nilpotent if $N^{k}=0$ for some positive integer $k$, where $N^{k}$ means the product of $N, k$ times;

(ii) An element $m$ of $M$ is called nilpotent if $m^{k}=0$ for some positive integer $k$.

The set of all nilpotent elements of $M$ is denoted by $N_{M}$. 
Definition 4. Let $M$ be an $R$-module and $N$ be a submodule of $M$. Then, the radical of $N$ denoted by $M-\operatorname{rad}(N)$ or $r(N)$ is defined to be intersection of all prime submodules of $M$ containing $N$. If $N$ is not contained in any prime submodule of $M$, then $M-\operatorname{rad}(N)=M$.

Theorem 7. [6, Theorem 3.13] Let $N$ be a submodule of a multiplication $R$ module $M$. Then $M-\operatorname{rad}(N)=\left\{m \in M: m^{k} \subseteq N\right.$ for some $\left.k>0\right\}$.

Corollary 8. Let $M$ be a multiplication $R$-module. Then $N_{M}$ is the intersection of all prime submodules of $M\left(r(0)=N_{M}\right)$.

Definition 5. A proper submodule $N$ of a module $M$ over a commutative ring $R$ is said to be weakly prime submodule if whenever $0 \neq r m \in N$, for some $r \in R, m \in M$, then $m \in N$ or $r \in(N: M)$.

Clearly, every prime submodule of a module is weakly prime submodule. However, since 0 is always weakly prime(by definition), a weakly prime submodule need not be prime.

Theorem 9. Let $N$ be a weakly prime submodule of $M$. If $(N: M) N \neq 0$, then $N$ is a prime submodule of $M$.

Proof. Suppose that $(N: M) N \neq 0$; we show that $N$ is prime. Let $r m \in N$. If $r m \neq 0$, then $N$ weakly prime gives $m \in N$ or $r \in(N: M)$. So assume that $r m=0$.First suppose that $r N \neq 0$, say $r n_{0} \neq 0$ where $n_{0} \in N$. Then $0 \neq r n_{0}=r\left(m+n_{0}\right) \in N$, so $r \in(N: M)$ or $m+n_{0} \in N$. Hence $r \in(N: M)$ or $m \in N$.So we can assume that $r N=0$. Next suppose that $m(N: M) \neq$ $0, m k_{0} \neq 0$ where $k_{0} \in(N: M)$. Then $0 \neq m k_{0}=\left(r+k_{0}\right) m \in N$. Therefore $m \in N$ or $r+k_{0} \in(N: M)$. Then $m \in N$ or $r \in(N: M)$. So we can assume that $m(N: M)=0$.

Since $(N: M) N \neq 0$, there exists $k \in(N: M)$ and $n \in N$ with $k n \neq 0$. Then $0 \neq k n=(r+k)(m+n) \in N ; r+k \in(N: M)$ or $m+n \in N$. Hence $r \in(N: M)$ or $m \in N$. So $N$ is prime submodule of $M$.

Compare the following Theorem with Theorem 1 in [1] .

Theorem 10. Let $M$ be a multiplication $R$-module.Let $N$ be a weakly prime submodule of $M$.If $N$ is not prime, $N^{2}=0$.

Proof. Since $M$ is a multiplication module, $N=(N: M) M$. Therefore, $N^{2}=$ $(N: M)^{2} M=(N: M) N=0$ by Theorem 9 .

Corollary 11. Let $M$ be a multiplication $R$-module and $N$ be weakly prime submodule of $M$. Then $N \subseteq r(0)$ or $r(0) \subseteq N$. 
Proof. If $N$ is a prime submodule, $r(0) \subseteq N$ by Corollary 8 . If $N$ is not prime submodule, $N \subseteq r(0)$ by Theorem 10 .

Recall that an ideal $I$ in a commutative ring $R$ is called semiprime if $r^{n} \in I$ for some $n \in Z^{+}$implies that $r \in I$. It is well known that an ideal $I$ is semiprime if and only if $I=\sqrt{I}=\left\{r \in R: r^{n} \in I\right.$ for some $\left.n \in Z^{+}\right\}$. A submodule $N$ of $M$ is called semiprime if $r^{n} m \in N$ for some $n \in Z^{+}$implies that $r m \in N$. It is clear that $N$ is semiprime if and only if $I^{n} V \subseteq N$ for some $n \in Z^{+}$implies that $I V \subseteq N$.

Theorem 12. Let $N$ be a proper submodule of a multiplication module $M$. Then $N$ is semiprime if and only if $U^{n} \subseteq N$ implies that $U \subseteq N$ for each submodule $U$ of $M$.

Proof. Let $N$ be a semiprime submodule and $U^{n} \subseteq N$ for some submodule $U$ of $M$. Suppose that $I$ be a presentation of $U$. Then $U^{n}=I^{n} M \subseteq N$. Therefore, $I^{n} \subseteq(N: M)$. Since $N$ is a semiprime submodule of $M,(N: M)$ is a semiprime ideal of $R$. Therefore, $I \subseteq(N: M)$ and so $U=I M \subseteq N$.

Conversely, let $U^{n} \subseteq N$ for some $n \in Z^{+}$implies that $U \subseteq N$ for any submodule $U$ of $M$.Let $I^{n} V \subseteq N$ for some ideal $I$ of $R$ and a submodule $V$ of $M$. Suppose that $J$ be a presentation of $V$. Therefore, $I^{n} V=I^{n} J M \subseteq N$ and so $(I V)^{n}=(I J)^{n} M \subseteq N$. Then $I V \subseteq N$.

Corollary 13. Let $N$ be a proper submodule of $M$. Then $N$ is semiprime if and only if $m^{n} \subseteq N$ for some $n \in Z^{+}$implies that $m \in N$ for every $m \in M$.

Proof. Let $N$ be a semiprime submodule. It is clear that $m^{n} \subseteq N$ for some $n \in Z^{+}$implies that $m \in N$ for every $m \in M$. Conversely, let $U \nsubseteq N$.Thus, there is $u \in U \backslash N$. Then $u^{n} \nsubseteq N$. Therefore $U^{n} \nsubseteq N$. Thus, $N$ is semiprime.

If $N$ is a submodule of $M$ such that $N$ is an intersection of prime submodules of $M$, then $N$ is semiprime submodule of $M$. We don't know if the converse is true in general, but it is true but it is true in the following special case(see, 3)

Theorem 14. Let $M$ be a multiplication $R$-module. Then, $N$ is a semiprime submodule of $M$ if and only if $r(N)=N$.

Proof. It is clear that $N \subseteq r(N)$ for any submodule $N$ of $M$. Let $N$ be a semiprime submodule of $M$. Let $m \in r(N)$. Then $m^{k} \subseteq N$ for some $k \in Z^{+}$. Since $N$ is semiprime, $m \in N$.Therefore, $N=r(N)$.

Conversely, let $N=r(N)$.Let $m^{n} \subseteq N$ for some $n \in Z^{+}$. Since $N=r(N)$, $m \in N$.Therefore, $N$ is semiprime submodule of $M$. 


\section{REFERENCES}

[1] Anderson, D.D.; Smith, Eric, Weakly prime ideals, Houston J. Math., 29, No.4, 831-840 (2003).

[2] C.P.Lu, Prime submodules of modules, Comment. Math. Univ. St. Paul., 33 (1984), no.1, $61-69$.

[3] Dauns, J., Prime modules, J.Reine Angew.Math.,298 (1978) , 156 - 181.

[4] Elbast.Z., Smith,P.F., Multiplication modules, Comm. Algebra, 16 (4), $1988,755-779$.

[5] Jenkins,J., Smith,P.F, On the Prime radical of a module over a commutative ring, Comm. Algebra 20, 1992,3593 - 3602.

[6] R.Ameri, On the prime submodules of multiplication modules, International journal of Mathematics and mathematical Sciences, 2003, 27, $1715-1725$.

[7] S.Ebrahimi Atani, Multiplication modules and related results, Archivum Mathematicum, 2004, 40, $407-414$.

[8] S.Ebrahimi Atani, The product of Multiplication submodules, Honam Mathematical Journal, 2005,no.1, 1-8.

[9] S.Ebrahimi Atani, F.Farzilipour, On Prime and Primary Submodules, Chiang. mai.J.Sci., 32 (1) , 2005, 5-9.

Received: September 18, 2006 